\title{
Editorial
}

\section{Plethysmography variability index and pre-cardiopulmonary bypass phlebotomy in children: Ideal physiology and clinical practice}

\author{
Seth Manoach* \\ Department of Medicine, Division of Pulmonary and Critical Care Medicine, Weill Cornell Medical College, \\ $N Y, U S A$
}

Received 29 August 2014

Accepted 30 August 2014

\section{Plethysmography variability index (PVI) and pre-cardiopulmonary bypass phlebotomy in children: What a supposedly flawed and negative study can teach about the physiology of "volume responsiveness" and assessment of shock}

Respiratory cycle-dependent dynamic volume status indices such as stroke volume variation ( $\Delta$ respSV), pulse pressure variation $(\triangle$ respPP), pulse oximetry plethysmography, and PVI perform best when tidal volume is $\geq 8 \mathrm{cc} / \mathrm{kg}$ and patient-ventilator interaction is extinguished [1]. In this issue of the Journal of Pediatric Intensive Care, Schloss et al. [2] employ these ventilatory parameters in their study of PVI. As interest in respiratory-cycled dynamic indices has grown, two data streams have pulled practice away from conditions that favor these measures. Robust prospective and historical data suggests that adults and children who are critically ill or undergoing major surgery benefit from

${ }^{*}$ Corresponding author: Dr. Seth Manoach, MD, CHCQM, FCCP, Department of Medicine, Division of Pulmonary and Critical Care Medicine, Weill Cornell Medical College, 1300 York Avenue, New York, NY 10065, USA. Tel.: +1 212746 7498; Fax: +1 646 9620406; E-mail: sem9030@med.cornell.edu. low tidal volume [3-5]. In the intensive care unit (ICU) patients derive neuropsychiatric and neuromuscular benefit that outweighs cardiopulmonary risk when they receive lighter sedation or analgo-sedation that preserves some patient-ventilator interaction [6-9]. This is true for all but the sickest patients with acute respiratory distress syndrome [10], and others with severe, uncontrolled obstructive airways disease, cardiogenic shock, status epilepticus, elevated intracranial pressure, and a few other illnesses. In pediatric [11] and adult critical care [3-9] evidence-based practice has moved so far from ideal conditions for obtaining accurate respiratory-cycled dynamic index data that the information would have to yield otherwise unavailable, consistent, high-impact patient-centered outcome benefits to justify routine clinical use.

2. Does Schloss et al. [2] help us determine whether dynamic respiratory cycled indices are worth changing our practice for children or adults?

A flurry of optimistic reports and reviews heralded the introduction of dynamic respiratory cycle- 
dependent indices $[1,12,13]$. The first of these closely followed a series of overly pessimistic assessments of traditional hemodynamic indices, such as central venous pressure (CVP) and pulmonary artery occlusion pressure (PAOP) [14-18]. A subsequent series of studies with adults, partially overlapping, but mostly conducted after the first group, failed to reproduce the robust correlations between dynamic index cut-off values and volume responsive improvements in cardiac performance. The latter studies generally included patients who had been excluded from the former series, but who manifest conditions commonly seen in ICU [19-24] and high-risk operative practice [25]. In contrast to earlier work [1], investigators who conducted the latter group of studies tended to treat patients as they would have been treated under normal conditions $[19,20]$. This is perhaps because of the lag time between study design and publication, during which the benefits of lung-protective ventilation and lighter analgo-sedation regimes became widely accepted. Likely, because of these changes in patient selection and treatment protocols, the area under the receiver operator curves for respiratory-cycled dynamic indices in the latter studies more closely resembled those of classical "static" indices like CVP and PAOP [19-23]. As a recent multi-center ICU prevalence study showed, only $18 \%$ of patients met the demanding conditions that dynamic indices require to reliably predict what is termed "volume responsiveness" [24].

PVI is a non-invasive plethysmography-based derivative of $\Delta$ respPP. The technique measures respiratory cycle flux in the perfusion index (PI). The PI is the ratio of pulsatile arterial to non-pulsatile light wavelengths reflected back to a pulse oximeter, and is expressed as a percentage. Because arterial perfusion generally falls in patients who are volume depleted or in other shock states, PI should fall as well. As explained below, in such patients this ratio should increase with mechanical inspiration and decrease with mechanical expiration. PVI is calculated by subtracting the minimum from maximum PI values and dividing by the maximum [26]. The greater the difference over the respiratory cycle, the greater the PVI. Because PVI does not require an arterial catheter, it is especially appealing in children.

Schloss et al. [2] measured PVI in a cohort of 31 North American children with a variety of congenital cardiac anomalies before and after they underwent therapeutic pre-cardiopulmonary bypass phlebotomy. They recorded baseline PVI after performing induc- tion, initiating positive pressure ventilation, and stabilizing the children on sevoflurane. They repeated this measurement after performing phlebotomy but prior to cardiopulmonary bypass. Although the authors carefully controlled ventilation, they resuscitated children who manifested signs of shock with variable doses of volume and pressors. Since this was a cohort of children undergoing cardiac surgery, a significant number had right heart failure and/or pulmonary hypertension. As the authors' state:

While this clinical situation does not allow us to test fluid responsiveness per se, it does give us the opportunity to observe PVI changes under conditions of acute intravascular volume changes associated with intraoperative phlebotomy [2].

These limitations are important, and whatever the authors' feelings about this supposed flaw, the paper is invaluable because of, and not despite it. Instead of having to extrapolate from adult data or rely on pediatric studies from poor nations with a high infectious disease burden and radically different medical infrastructure [27], Schloss et al. [2] give us direct evidence that respiratory-cycled dynamic indices fail when practicing clinicians have to take care of children who are critically ill and develop peri-operative shock. Like those clinicians in Schloss et al. [2], we also treat our sickest patients with rapid and simultaneous interventions like vasopressors and fluid, which can, in combination, undermine the accuracy of dynamic indices. As a result, Schloss et al.'s [2] "wild type" data is very pertinent to pediatric and adult intensivists, anesthesiologists, surgeons, and emergency physicians.

\section{Principal finding: PVI did not detect a shock state}

Schloss et al. [2] found that PVI did not detect shock (or "volume responsiveness") triggered by phlebotomy in this cohort of children, while classic measures like falls in systolic blood pressure (SBP) $(P=0.001)$ and mean arterial pressure (MAP) $(P=0.017)$ did, as did near infrared spectroscopy (NIRS). NIRS detected a statistically significant reduction in cerebral perfusion (cerebral oximetry fell 76 to $68 \%, P=0.005$ ). Although pre-and post-phlebotomy PVI failed to detect shock $(P=0.55)$, it did predict total volume administration. The latter finding is a step removed from the primary physiologic changes revealed by SBP, MAP, and NIRS. 
Although critics might be tempted to dismiss the study because resuscitation between the two PVI measurements limited the net volume and blood loss [2], this did not prevent the cohort of children from experiencing shock state sufficient to be detected by falls in systolic blood pressure, mean arterial pressure, and perhaps most important, cerebral perfusion. The fall in cerebral oximetry detected by NIRS is notable because sevoflurane tends to maintain cerebral blood flow without significantly changing cerebral metabolic rate of oxygen consumption [28].

\section{Physiology and failure of dynamic respiratory cycled indices: $\Delta_{\text {resp }} S V, \Delta_{\text {resp }} P P$ and the special case of PVI}

Under optimal conditions, dynamic respiratory cycle dependent-indices are thought to detect volume responsiveness when patients are on the ascending, or volume responsive limb of the left ventricular Starling curve. In classical physiologic terms [29, 30] these indices can be understood by following the interaction between Guyton venous return curves and Starling ventricular performance curves over the respiratory cycle. Those not familiar with Guyton venous return curves and their limitations [31] can understand dynamic respiratory cycled indices by imagining points instead of lines moving up and down along the more familiar Starling curve as intrathoracic pressure changes.

During mechanical insufflation, the venous return curve shifts downward and "leftward," intersecting the ascending limb of the right ventricular Starling curve at a lower point along the y-axis, reflecting decreased right ventricular filling and cardiac output. This occurs because increased intrathoracic pressure reduces the pressure gradient between stressed venous volume and the right ventricle (RV), and because inflation of the lung puts direct pressure on vessels or chambers through or into which venous blood returns to the RV.

During mechanical expiration, intra-thoracic pressure falls, and the venous return curve shifts back upward and rightward in parallel, intersecting the right ventricular Starling curve at a higher point. This is physiologically similar to administering a fluid bolus. In plain language, respiratory cycle-related pressure flux has the effect of subtracting and adding back small fluid boluses to the right heart in inverse proportion to changes in intrathoracic pressure. The opposite filling pattern occurs during normal negative pressure breathing, though usually to a lesser degree.

Over approximately three-four heart beats blood transverses the pulmonary vasculature, resulting in respiratory phase-shifted differential left ventricular filling. If the respiratory rate remains sufficiently low relative to the heart rate $(<1: 3.6)$ [32], the relatively greater venous return to the RV during mechanical expiration is delivered to the left ventricle (LV) during mechanical inspiration. With normal RV function and pulmonary vascular resistance, the right ventricular starling curve is nearly vertical over the range of expected points of intersection with the venous return curve, so adding or subtracting venous return to the $\mathrm{RV}$ decreases or increases right ventricular output in a nearly $1: 1$ ratio. As a result, the right ventricular starling curve can be, and in most representations is, abstracted out of the model [29].

If the points of intersection between the venous return curve and the left ventricular Starling curve during mechanical expiration and inspiration both lie along the ascending, or preload-responsive portion, more blood is ejected into the systemic circulation during mechanical inspiration, and this is detected as an increase in pulse pressure, LV stroke volume, or perfusion index. Patients in whom respiratory-cycled transient loading and unloading of the RV result in sufficiently large changes in left ventricular stroke volume are thought to be "volume responsive." In these patients, $\Delta_{\text {respSV }}, \Delta_{\text {respPP }}$ or PVI are typically above $10-15 \%$, and the transient loading and unloading of the $\mathrm{RV}$ reflected by these indices predicts an increase in left sided cardiac performance in response to an exogenous fluid bolus. The development of many of the concepts underlying dynamic respiratory cycle indices are explained succinctly in other publications [15, 29, 30].

As noted above, Schloss et al. [2] were unable to standardize volume replacement. Volume repletion replaces volume loss, which decreases PVI. But, as Schloss et al. [2] point, out, the children were unlikely to have been sufficiently resuscitated to reverse their shock state, as SBP, MAP, cerebral oxygenation, and perfusion index all fell after phlebotomy [2].

The PVI-altering effects of variable pressor administration in this study are unlikely to explain the failure of PVI because only three of 31 children received single doses of phenylephrine. To the extent that phenylephrine affected the outcome, the net effect was probably to dampen PVI, as it would dampen other respiratory-cycled dynamic indices. Because 
PVI depends on detecting microvascular perfusion changes that are extremely sensitive to endogenous or exogenous catecholamines, PVI is more likely than $\Delta$ respSV, or $\Delta$ respPP to fail as a consequence of circulatory failure, noxious stimuli or treatment with vasopressors [26, 33-35].

Phenylephrine's pure $\alpha-1$ agonist properties recruit unstressed volume from venous capacitance vessels to stressed volume, raising mean systemic filling pressure and shifting the venous return curve upward and rightward. Phenylephrine also increases pulmonary and systemic afterload, rotating the venous return curve counter-clockwise (down along the y axis) so the venous return and starling curves intersect at a lower cardiac output (CO) point. To the degree that increasing afterload also shifts the Starling curve down and to the right, the intersection of the two curves would occur at an even lower venous return/cardiac output point, and at a higher CVP. Increased filling pressures and afterload decrease the relative effect of respiratory cycle-related flux in intrathoracic pressure, dampening any dynamic respiratory cycle-dependent index. The net effect of phenylephrine on cardiac output is generally thought to be negative, but this, and the drug's effect on organ perfusion, depend on baseline hemodynamics.

Epinephrine [11] and norepinephrine [36], which are more commonly used in the ICU than phenylephrine for septic and cardiogenic shock in children [11] and adults [36], generally shift the venous return curve up and to the right by recruiting unstressed volume from venous capacitance into stressed volume that augments venous return $[29,30,37,38]$. The opposing effects of these agents on afterload and cardiac performance tend to cancel each other out, so $\mathrm{CO}$ generally increases with a higher mean systemic filling pressure that dampens the relative effect of any given cyclic change in intrathoracic pressure. As a result, these agents dampen respiratory cycledependent indices [37, 38]. As many patients with high dynamic respiratory indices may be "pressor responsive" $[29,37,38]$ dynamic index values cannot be said to uniquely diagnose "volume responsiveness" but "shock resulting from inadequate venous return." By recruiting blood from venous capacitance vessels and increasing both venous return and mean systemic filling pressures, these agents correct what may appear to a dynamic index to be a volume deficit. In extreme cases, for example, in anaphylactic shock, dynamic indices may appear to signal large fluid deficits instead of fluid shifts from stressed to unstressed volume [29]. Although fluid boluses may initially be lifesaving, too much volume increases pulmonary, airway, and tissue edema. Ultimately, what saves lives in anaphylactic shock is not fluid, but exogenous epinephrine.

Schloss et al. [2] mention that RV failure or increased pulmonary vascular resistance may have played a role in the failure of PVI to detect a pre- to post phlebotomy shock state. With pulmonary hypertension, the RV Starling curve is stably shifted rightward and down so increasing venous return can no longer be assumed to directly increase LV preload and performance. The RV starling curve may transiently shift even further down and to the right during mechanical insufflation as vascular resistance to any given $\mathrm{RV}$ impulse increases. The venous return curve rotates counterclockwise to a lower venous return/cardiac output point, because cardiac output falls at any given mean systemic filling pressure. The net result is that the two curves intersect at a far lower venous return/cardiac output point than they would with normal right-sided hemodynamics. Afterload-exaggerated falls in RV stroke volume during mechanical insufflation are phase-shifted to the LV, exaggerating the fall in SV, PP, and PI during mechanical expiration. This in turn raises $\Delta$ respSV, $\Delta$ respPP and PVI, suggesting "volume responsiveness". In fact, adding volume is likely to increase RV afterload and/or cause the interventricular septum to bow into the LV cavity, lowering CO [21]. Such false positive [21, 22] dynamic index values can harm patients. Because of this, Mahjoub et al. [22] suggest coupling echocardiography with respiratory-cycled dynamic indices to screen out patients with RV failure/pulmonary hypertension.

The "limitations" of Schloss et al.'s [2] pediatric study, with related adult literature, demonstrate what follows from Guyton and Starling [29, 30]: dynamic respiratory cycle-dependent indices only predict volume responsiveness to the degree that vasomotor tone remains constant and there is an uninterrupted, physiologically "normal" connection between RV and LV preload. These papers highlight the role of catecholamines, cytokines and medications that alter vasomotor tone $[2,29,37,38]$. They also highlight the importance of RV dysfunction, elevated PVR [2, 21, 22], low respiratory system compliance [23], high intrathoracic pressure [20], dysrhythmia, and high respiratory to heart rate ratios [32] that disrupt transpulmonary blood flow. Ongoing volumeindependent changes in cardiac performance, PVR, 
and systemic vasomotor tone are typical of adults and children [39] sick enough to need intensive care [24] or cardiac surgery [25]. Ideal conditions for dynamic respiratory-cycled monitors are the exception, and not the rule in our day-to-day clinical practice [24, 25].

\section{Alternative methods of assessing "volume responsiveness"}

Although not the first work to study passive leg raise (PLR) [40], Marik et al.'s [41] 2011 work has received much recent attention. Marik et al. [41] demonstrated that PLR was superior to $\triangle$ respSV for predicting an increase in stroke volume index. Because children have smaller limbs than adults, relative to the size of their torso and head, the impact of passive leg raise is unlikely to be as significant in infants and young children as in older children and adults. PLR is only sufficiently sensitive if correlated to changes in cardiac output measured by invasive or potentially unreliable techniques such as thermodilution, bioreactance, or aortic Doppler [40-43]. PLR performs better than dynamic respiratory-cycled indices when there is low respiratory system compliance, and is not dependent on tidal volume, cardiac rhythm, or respiratory to heart rate ratios [40]. As with respiratory-cycled dynamic indices, a positive response to PLR signals only that mean systemic filling pressure may be pressor or volume responsive [42]. A negative test can mean that a patient is volume replete, or as is the case even in mild intra-abdominal hypertension, that there is increased resistance to venous return [43].

Finally, because of the problems with respiratorycycled and postural dynamic predictors of "shock resulting from inadequate venous return" [29] we have moved back to where we started - giving small fluid boluses, and assessing whether an easily accessible measure, such as the resulting change in pulse pressure, can reliably predict a change in cardiac output. One such trial, conducted under controlled conditions similar to the earliest $\Delta$ respPP trials, found a weak but positive correlation between the two [44].

\section{Where does this leave us?}

Absent from most dynamic "volume responsiveness" literature is a discussion of whether it matters if we correct putative volume deficits that can be found only under tight physiologic constraints and interpreted without reference to available downstream (e.g. lactate, mixed central venous oxygen saturation, and mixed venous oxygen saturation) [15] or bedside data [45-47]. Astute clinicians watch trends, not discrete cut-off points, correlate their findings with clinical signs and symptoms, and continuously re-evaluate the effect of ongoing clinical strategies [45-52]. The Fluids and Catheters Treatment Trial is one of the few recent studies to demonstrate that careful volume assessment could yield a patient-centered benefit: early liberation from mechanical ventilation. The investigators did this by following trends in either CVP or PAOP, and using these and other clinical data to limit extravascular lung water in acute respiratory distress syndrome patients [49]. Although no study reliably establishes a fixed point that predicts volume responsiveness for CVP or PAOP, multiple trials show that across study populations, these values rise with fluid administration and fall with diuresis [49-52].

The negative primary outcome Schloss et al. [2] found in their methodologically flawed, but clinically important study matters because it adds North American pediatric data to the growing mass of adult studies questioning the value of dynamic respiratory-cycled indices in "wild type" clinical settings. Anesthesiologists in Schloss et al. [2] acted to correct shock as we and other well-intended providers would have when changes in SBP, MAP and NIRS hinted at danger. Their literature review reminds us that when patients develop severe shock, we may be able to detect significant changes in pulse oximetry plethysmography without proprietary technology [53]. Even without imposing the tight constraints needed to obtain publication-ready cut-off values for dynamic indices, astute clinicians may be able to identify shock by carefully watching respiratory cycled pulse oximetry and invasive arterial tracings on a bedside monitor [15, 54]. Any data point or trend may be useful, but only if we understand its underlying physiology and integrate that with the stream of clinical information that is constantly generated at the bedside.

\section{References}

[1] Michard F, Boussat S, Chemla D, Anguel N, Mercat A, Lecarpentier Y, et al. Relation between respiratory changes in arterial pulse pressure and fluid responsiveness in septic patients with acute circulatory failure. Am J Respir Crit Care Med 2000;162(1):134-8. 
[2] Schloss B, Naguib A, Bissonnette B, Winch P, Rice J, Galantowicz $\mathrm{M}$, et al. Plethysmography variability index response to isovolemic hemodilution in children prior to surgery for congenital heart disease. J Pediatr Intensive Care 2014;3(1): 35-40.

[3] Ventilation with lower tidal volumes as compared with traditional tidal volumes for acute lung injury and the acute respiratory distress syndrome. The Acute Respiratory Distress Syndrome Network. N Engl J Med 2000;342(18):1301-8.

[4] Gajic O, Frutos-Vivar F, Esteban A, Hubmayr RD, Anzueto A. Ventilator settings as a risk factor for acute respiratory distress syndrome in mechanically ventilated patients. Intensive Care Med 2005;31(7):922-6.

[5] Futier E, Constantin JM, Paugam-Burtz C, Pascal J, Eurin M, Neuschwander A, et al. IMPROVE Study Group. A trial of intraoperative low-tidal-volume ventilation in abdominal surgery. N Engl J Med 2013;369(5):428-37.

[6] Levine S, Nguyen T, Taylor N, Friscia ME, Budak MT, Rothenberg $\mathrm{P}$, et al. Rapid disuse atrophy of diaphragm fibers in mechanically ventilated humans. N Engl J Med 2008;358(13):1327-35.

[7] Kress JP, Pohlman AS, O' Connor MF, Hall JB. Daily interruption of sedative infusions in critically ill patients undergoing mechanical ventilation. N Engl J Med 2000;342(20):1471-7.

[8] Devabhakthuni S, Armahizer MJ, Dasta JF, Kane-Gill SL. Analgosedation: A paradigm shift in intensive care unit sedation practice. Ann Pharmacother 2012;46(4):530-40.

[9] Schweickert WD, Pohlman MC, Pohlman AS, Nigos C, Pawlik AJ, Esbrook CL, et al. Early physical and occupational therapy in mechanically ventilated, critically ill patients: A randomised controlled trial. Lancet 2009;373(9678): 1874-82.

[10] Papazian L, Forel JM, Gacouin A, Penot-Ragon C, Perrin G, Loundou A, et al.; ACURASYS Study Investigators. Neuromuscular blockers in early acute respiratory distress syndrome. N Engl J Med. 2010;363(12):1107-16.

[11] Kissoon N, Carcillo JA, Espinosa V, Argent A, Devictor D, Madden M, et al.; Global Sepsis Initiative Vanguard Center Contributors. World Federation of Pediatric Intensive Care and Critical Care Societies: Global Sepsis Initiative. Pediatr Crit Care Med 2011;12(5):494-503.

[12] Cannesson M, Slieker J, Desebbe O, Bauer C, Chiari P, Hénaine R, et al. The ability of a novel algorithm for automatic estimation of the respiratory variations in arterial pulse pressure to monitor fluid responsiveness in the operating room. Anesth Analg 2008;106(4):1195-200.

[13] Marik PE, Cavallazzi R, Vasu T, Hirani A. Dynamic changes in arterial waveform derived variables and fluid responsiveness in mechanically ventilated patients: A systematic review of the literature. Crit Care Med 2009;37(9):2642-7.

[14] Marik PE, Baram M, Vahid B. Does central venous pressure predict fluid responsiveness? A systematic review of the literature and the tale of seven mares. Chest 2008;134(1):172-8.

[15] Manoach S, Weingart SD, Charchaflieh J. The evolution and current use of invasive hemodynamic monitoring for predicting volume responsiveness during resuscitation, perioperative, and critical care. J Clin Anesth 2012;24(3):242-50.

[16] Murphy GS, Vender JS. Con: Is the pulmonary artery catheter dead? J Cardiothorac Vasc Anesth 2007;21(1):147-9.

[17] Vender JS. Pulmonary artery catheter utilization: The use, misuse, or abuse. J Cardiothorac Vasc Anesth 2006;20(3): 295-9.

[18] Friese RS, Shafi S, Gentilello LM. Pulmonary artery catheter use is associated with reduced mortality in severely injured patients: A National Trauma Data Bank analysis of 53,312 patients. Crit Care Med 2006;34(6):1597-601.

[19] De Backer D, Heenen S, Piagnerelli M, Koch M, Vincent JL. Pulse pressure variations to predict fluid responsiveness: Influence of tidal volume. Intensive Care Med 2005;31(4) 517-23.

[20] Lakhal K, Ehrmann S, Benzekri-Lefèvre D, Runge I, Legras A, Dequin PF, et al. Respiratory pulse pressure variation fails to predict fluid responsiveness in acute respiratory distress syndrome. Crit Care 2011;15(2):R85.

[21] Wyler von Ballmoos M, Takala J, Roeck M, Porta F, Tueller D, Ganter CC, et al. Pulse-pressure variation and hemodynamic response in patients with elevated pulmonary artery pressure: A clinical study. Crit Care 2010;14(3):R111.

[22] Mahjoub Y, Pila C, Friggeri A, Zogheib E, Lobjoie E, Tinturier $\mathrm{F}$, et al. Assessing fluid responsiveness in critically ill patients False-positive pulse pressure variation is detected by Doppler echocardiographic evaluation of the right ventricle. Crit Care Med 2009;37(9):2570-5.

[23] Monnet X, Bleibtreu A, Ferré A, Dres M, Gharbi R, Richard $\mathrm{C}$, et al. Passive leg-raising and end-expiratory occlusion tests perform better than pulse pressure variation in patients with low respiratory system compliance. Crit Care Med 2012;40(1):152-7.

[24] Mahjoub Y, Lejeune V, Muller L, Perbet S, Zieleskiewicz $\mathrm{L}$, Bart F, et al. Evaluation of pulse pressure variation validity criteria in critically ill patients: A prospective observational multicentre point-prevalence study. $\mathrm{Br} \mathrm{J}$ Anaesth 2014;112(4):681-5.

[25] Lansdorp B, Lemson J, van Putten MJ, de Keijzer A, van der Hoeven JG, Pickkers P. Dynamic indices do not predict volume responsiveness in routine clinical practice. $\mathrm{Br} \mathrm{J}$ Anaesth 2012;108(3):395-401.

[26] Fischer MO, Pelissier A, Bohadana D, Gérard JL, Hanouz JL, Fellahi JL. Prediction of responsiveness to an intravenous fluid challenge in patients after cardiac surgery with cardiopulmonary bypass: A comparison between arterial pulse pressure variation and digital plethysmographic variability index. J Cardiothorac Vasc Anesth 2013;27(6):1087-93.

[27] Oshima T, Karasawa F, Okazaki Y, Wada H, Satoh T. Effects of sevoflurane on cerebral blood flow and cerebral metabolic rate of oxygen in human beings: A comparison with isoflurane. Eur J Anaesthesiol 2003;20(7):543-7.

[28] Maitland K, Kiguli S, Opoka RO, Engoru C, Olupot-Olupot P, Akech SO, et al. FEAST Trial Group. Mortality after fluid bolus in African children with severe infection. N Engl J Med 2011;364(26):2483-95

[29] Feihl F, Broccard AF. Interactions between respiration and systemic hemodynamics. Part I: Basic concepts. Intensive Care Med 2009;35(1):45-54.

[30] Magder S. Bench-to-bedside review: An approach to hemodynamic monitoring -Guyton at the bedside. Crit Care 2012;16(5):236.

[31] Beard DA, Feigl EO. Understanding Guyton's venous return curves. Am J Physiol Heart Circ Physiol 2011;301(3):H629 33.

[32] De Backer D, Taccone FS, Holsten R, Ibrahimi F, Vincent $\mathrm{JL}$. Influence of respiratory rate on stroke volume variation in mechanically ventilated patients. Anesthesiology 2009; 110(5):1092-7.

[33] Takeyama M, Matsunaga A, Kakihana Y, Masuda M, Kuniyoshi T, Kanmura Y. Impact of skin incision on the pleth variability index. J Clin Monit Comput 2011;25(4):215-21. 
[34] Biais M, Cottenceau V, Petit L, Masson F, Cochard JF, Sztark F. Impact of norepinephrine on the relationship between pleth variability index and pulse pressure variations in ICU adult patients. Crit Care 2011;15(4):R168.

[35] Monnet X, Guérin L, Jozwiak M, Bataille A, Julien F, Richard $\mathrm{C}$, et al. Pleth variability index is a weak predictor of fluid responsiveness in patients receiving norepinephrine. $\mathrm{Br} \mathrm{J}$ Anaesth 2013;110(2):207-13.

[36] De Backer D, Biston P, Devriendt J, Madl C, Chochrad D, Aldecoa C, et al. SOAP II Investigators. Comparison of dopamine and norepinephrine in the treatment of shock. N Engl J Med 2010;362(9):779-89.

[37] Maas JJ, Pinsky MR, de Wilde RB, de Jonge E, Jansen JR. Cardiac output response to norepinephrine in postoperative cardiac surgery patients: Interpretation with venous return and cardiac function curves. Crit Care Med 2013;41(1):143-50.

[38] Persichini R, Silva S, Teboul JL, Jozwiak M, Chemla D, Richard C, Monnet X. Effects of norepinephrine on mean systemic pressure and venous return in human septic shock. Crit Care Med 2012;40(12):3146-53

[39] Fenton KE, Sable CA, Bell MJ, Patel KM, Berger JT. Increases in serum levels of troponin I are associated with cardiac dysfunction and disease severity in pediatric patients with septic shock. Pediatr Crit Care Med 2004;5(6):533-8.

[40] Monnet X, Rienzo M, Osman D, Anguel N, Richard C, Pinsky $\mathrm{MR}$, et al. Passive leg raising predicts fluid responsiveness in the critically ill. Crit Care Med 2006;34(5):1402-7.

[41] Marik PE, Levitov A, Young A, Andrews L. The use of bioreactance and carotid Doppler to determine volume responsiveness and blood flow redistribution following passive leg raising in hemodynamically unstable patients. Chest 2013;143(2):364-70

[42] Monnet X, Jabot J, Maizel J, Richard C, Teboul JL. Norepinephrine increases cardiac preload and reduces preload dependency assessed by passive leg raising in septic shock patients. Crit Care Med 2011;39(4):689-94

[43] Mahjoub Y, Touzeau J, Airapetian N, Lorne E, Hijazi M, Zogheib E, et al. The passive leg-raising maneuver cannot accurately predict fluid responsiveness in patients with intraabdominal hypertension. Crit Care Med 2010;38(9):1824-9.
[44] Lakhal K, Ehrmann S, Perrotin D, Wolff M, Boulain T. Fluid challenge: Tracking changes in cardiac output with blood pressure monitoring (invasive or non-invasive). Intensive Care Med 2013;39(11):1953-62.

[45] Nohria A, Tsang SW, Fang JC, Lewis EF, Jarcho JA, Mudge $\mathrm{GH}$, et al. Clinical assessment identifies hemodynamic profiles that predict outcomes in patients admitted with heart failure. J Am Coll Cardiol 2003;41(10):1797-804.

[46] Vazquez R, Gheorghe C, Kaufman D, Manthous CA. Accuracy of bedside physical examination in distinguishing categories of shock: A pilot study. J Hosp Med 2010;5(8):471-4.

[47] Horeczko T, Enriquez B, McGrath NE, Gausche-Hill M, Lewis RJ. The Pediatric Assessment Triangle: Accuracy of its application by nurses in the triage of children. J Emerg Nurs 2013;39(2):182-9.

[48] Robin E, Costecalde M, Lebuffe G, Vallet B. Clinical relevance of data from the pulmonary artery catheter. Critical Care 2006:10(Suppl 3):S3.

[49] National Heart, Lung, and Blood Institute Acute Respiratory Distress Syndrome Clinical Trials Network, Wiedemann HP, Wheeler AP, Bernard GR, Thompson BT, Hayden D, deBoisblanc B, et al. Comparison of two fluid-management strategies in acute lung injury. N Engl J Med 2006;354(24):2564-75.

[50] Crexells C, Chatterjee K, Forrester JS, Dikshit K, Swan HJ. Optimal level of filling pressure in the left side of the heart in acute myocardial infarction. N Engl J Med 1973;289(24): 1263-6.

[51] Rivers E, Nguyen B, Havstad S, Ressler J, Muzzin A, Knoblich B, et al. Early Goal-Directed Therapy Collaborative Group. Early goal-directed therapy in the treatment of severe sepsis and septic shock. N Engl J Med 2001;345(19):1368-77.

[52] Drazner MH, Hamilton MA, Fonarow G, Creaser J, Flavell C, Stevenson LW. Relationship between right and left-sided filling pressures in 1000 patients with advanced heart failure. J Heart Lung Transplant 1999;18(11):1126-32.

[53] Shamir M, Eidelman LA, Floman Y, Kaplan L, Pizov R. Pulse oximetry plethysmographic waveform during changes in blood volume. Br J Anaesth 1999;82(2):178-81.

[54] Rick JJ, Burke SS. Respirator paradox. South Med J 1978;71(11):1376-8, 1382. 